\title{
Epitaxial $\mathrm{YBa}_{2} \mathrm{Cu}_{3} \mathrm{O}_{7}$ Films on Rolled-Textured Metals for High- Temperature Superconducting Applications
}

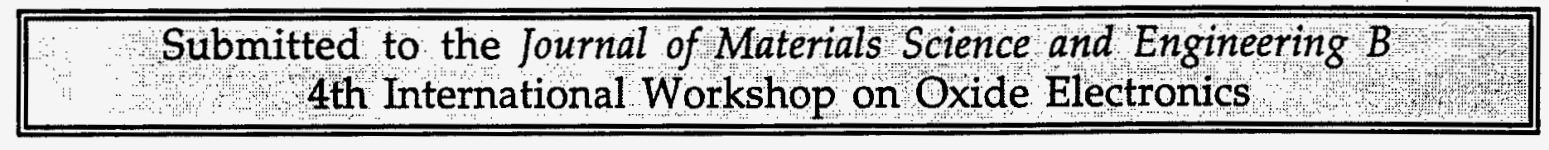

D. P. Norton, C. Park, C. Prouteau, D. K. Christen,

M. F. Chisholm, J. D. Budai

Solid State Division,

Oak Ridge National Laboratory,

P. O. Box 2008, Oak Ridge, TN 37831-6056

A. Goyal, E. Y. Sun, D. F. Lee, D. M. Kroeger, E. Specht

Metals and Ceramics Division,

Oak Ridge National Laboratory,

P. O. Box 2008, Oak Ridge, TN 37831-6033

M. Paranthaman

Chemical and Analytical Sciences Division,

Oak Ridge National Laboratory,

P. O. Box 2008, Oak Ridge, TN 37831-6100
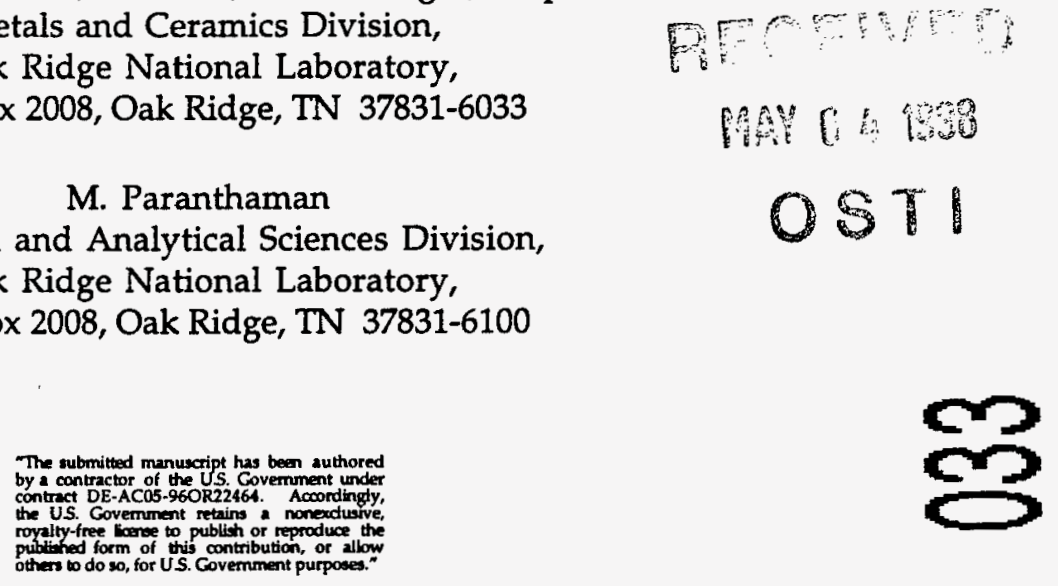

April 1998

Prepared by

Solid State Division

Oak Ridge National Laboratory

P.O. Box 2008

Oak Ridge, Tennessee 37831-6056

managed by

LOCKHEED MARTIN ENERGY RESEARCH CORP.

for the

U.S. DEPARTMENT OF ENERGY

under contract DE-AC05-96OR22464 


\section{DISCLAIMER}

This report was prepared as an account of work sponsored by an agency of the United States Government. Neither the United States Government nor any agency thereof, nor any of their employees, makes any warranty, express or implied, or assumes any legal liability or responsibility for the accuracy, completeness, or usefulness of any information, apparatus, product, or process disciosed, or represents that its use would not infringe privately owned rights. Reference herein to any specific commercial product, process, or service by trade name, trademark, manufacturer, or otherwise does not necessarily constitute or imply its endorsement, recommendation, or favoring by the United States Government or any agency thereof. The views and opinions of authors expressed herein do not necessarily state or reflect those of the United States Government or any agency thereof. 
Epitaxial $\mathrm{YBa}_{2} \mathrm{Cu}_{3} \mathrm{O}_{7}$ Films on Rolled-Textured Metals for High-Temperature Superconducting Applications

D. P. Norton, C. Park, C. Prouteau, D. K. Christen, M. F. Chisholm, J. D. Budai Solid State Division, Oak Ridge National Laboratory, P. O. Box 2008, Oak Ridge, TN 37831-6056

A. Goyal, E. Y. Sun, D. F. Lee, D. M. Kroeger, E. Specht Metals and Ceramics Division, Oak Ridge National Laboratory, P. O. Box 2008, Oak Ridge, TN 37831-6033

M. Paranthaman

Chemical and Analytical Sciences Division, Oak Ridge National Laboratory, P. O. Box 2008, Oak Ridge, TN 37831-6100 


\begin{abstract}
The epitaxial growth of high temperature superconducting (HTS) films on rolled-textured metal represents a viable approach for long-length superconducting tapes. Epitaxial, $0.5 \mu \mathrm{m}$ thick $\mathrm{YBa}_{2} \mathrm{Cu}_{3} \mathrm{O}_{7}(\mathrm{YBCO})$ films with critical current densities, $J_{c^{\prime}}$ greater than $1 \mathrm{MA} / \mathrm{cm}^{2}$ have been realized on rolled-textured (001) $\mathrm{Ni}$ tapes with yttria-stabilized zirconia (YSZ)/ $\mathrm{CeO}_{2}$ oxide buffer layers. This paper describes the synthesis using pulsed-laser deposition (PLD) of epitaxial oxide buffer layers on biaxially-textured metal that comprise the so-called rolling-assisted biaxially-textured substrates (RABiTS ${ }^{\mathrm{TM}}$ ). The properties of the buffer and $\mathrm{YBa}_{2} \mathrm{Cu}_{3} \mathrm{O}_{7}$ films on rolled-textured $\mathrm{Ni}$ are discussed, with emphasis given to the crystallographic and microstructural properties that determine the superconducting properties of these multilayer structures.
\end{abstract}

\title{
Introduction
}

Significant effort has been directed towards developing high-current superconducting wire technologies [1,2]. Much of this work has focused on achieving the crystallographic texture needed in HTS wire or tape architectures in order to realize high critical current densities at $77 \mathrm{~K}$. It has been recognized for some time that randomly oriented polycrystalline HTS materials have critical current densities, $J_{c^{\prime}}<500 \mathrm{~A} / \mathrm{cm}^{2}$. In contrast, oriented YBCO thin films grown epitaxially on single-crystal oxide substrates, such as (001) $\mathrm{SrTiO}_{3}$, exhibit $J_{c}$ values $>1 \mathrm{MA} / \mathrm{cm}^{2}$ at $77 \mathrm{~K}$ [3]. This large difference between randomly-oriented HTS ceramics and single crystal-like epitaxial 
films is directly related to the misorientation angles at the grain boundaries in polycrystalline materials. Values. for $J_{c}$ across a grain boundary decrease significantly as the misorientation angle increases, with weak-link behavior observed for grain boundary misorientation angles greater than $\sim 10^{\circ}[4]$. In order to achieve high critical current densities $\left(\sim 10^{6} \mathrm{~A} / \mathrm{cm}^{2}, 77 \mathrm{~K}\right)$, the HTS superconducting wire or tape must possess a high degree of both in-plane and out-of-plane grain alignment over the conductor's entire length. Preferably, this would be achieved with YBCO, because the limits for dissipation-free current at $77 \mathrm{~K}$ in an applied magnetic field are most favorable for this material $[5,6]$.

Two deposited HTS conductor approaches have recently received significant attention as a means of producing long-length HTS tapes possessing the in-plane and out-of-plane crystallographic texture necessary for high values of $J_{c^{\prime}}$. One technique, known as ion beam-assisted deposition (IBAD), utilizes an ion beam to induce in-plane alignment of a depositing film as is schematically illustrated in Fig. $1[7,8]$. Related techniques that also utilize energetic ions to induce crystallographic orientation in polycrystalline films include PLD with inclined substrates [9] and bias sputter deposition [10]. With these energetic ion-assisted approaches, only the film possesses significant crystallographic texture, with the crystallinity of the supporting substrate material assumed to be irrelevant. This technique offers significant flexibility in substrate choice, and has been shown effective for a variety of thin film and substrate materials. However, the necessity of an ion beam in 
processing long length conductors is generally viewed as a disadvantage. An alternative approach also shown in Fig. 1 involves the formation of crystallographic texture through mechanical deformation and subsequent recrystallization of a metal substrate (11). The deposition of appropriate epitaxial buffer layers on the biaxially-textured metal results in an oriented architecture known as a rolling-assisted biaxially-textured substrate $\left(\operatorname{RABiTS}^{\mathrm{TM}}\right)[12,13]$. The RABiTS ${ }^{\mathrm{TM}}$ approach is obviously restricted to metals for which advantageous crystallographic texture can be achieved through thermo-mechanical deformation. A key advantage of this approach is in the simplicity of producing the initial in-plane alignment required for high $J_{c}$ by a cold-rolling and annealing process that can be easily scaled to produce arbitrary substrate lengths. In this paper, we review the synthesis and properties of superconducting $\mathrm{RABiTS}^{\mathrm{TM}}$ using pulsed-laser deposition, and discuss recent microstructural characterization of these structures

\section{RABiTS $^{\mathrm{TM}}$ Process for HTS Conductors}

The specific implementation of the $\mathrm{RABiTS}^{\mathrm{TM}}$ approach that has produced HTS films with high values of $J_{c}$ utilizes a biaxially textured (001) Ni tape, formed by recrystallization of cold-rolled pure $\mathrm{Ni}[11]$, as the initial, in-plane aligned substrate. Smooth $\mathrm{Ni}$ tapes that are $25-125 \mu \mathrm{m}$ thick have been produced possessing a relatively sharp cube orientation with a FWHM out-ofplane texture $\Delta \theta \sim 6^{\circ}-10^{\circ}$ and an in-plane texture $\Delta \phi \sim 6^{\circ}-15^{\circ}$, depending on the specific rolling and annealing conditions, as well as material purity, with 
a grain size ranging from $\sim 30-100 \mu \mathrm{m}$ in diameter. A (001)-oriented oxide buffer layer architecture is then epitaxially grown that maintains the sharp crystallographic cube texture of the metal substrate while providing a barrier to chemical interaction with the Ni. Typically, the epitaxial growth of any (001)-oriented cubic oxide on a (001) Ni surface is inhibited by the formation of (111) $\mathrm{NiO}$ at the oxide/metal interface [14]. In order to suppress the formation of $\mathrm{NiO}$ and achieve (001)-oriented epitaxy directly on the (001) $\mathrm{Ni}$ surface, $\mathrm{H}_{2}$ gas is introduced into the PLD chamber during the initial stages of $\mathrm{CeO}_{2}$ growth. Hydrogen is effective in reducing $\mathrm{NiO}$, while having little effect on the $\mathrm{CeO}_{2}$ film. The nucleation of the $\mathrm{CeO}_{2}$ layer in the presence of $\mathrm{H}_{2}$ results in the formation of (001) $\mathrm{CeO}_{2}$, with subsequent growth in either vacuum or a background of oxygen maintaining this orientation. Similar results for $\mathrm{CeO}_{2}$ on (100) $\mathrm{Ni}$ have been achieved using both sputtering [15] and e-beam evaporation [16]. This (001)-oriented $\mathrm{CeO}_{2}$ layer provides an oxide template directly on the metal surface for the subsequent epitaxial growth of additional oxide buffer and HTS layers.

\section{Crystallographic Properties}

The local crystallographic texture for a $\mathrm{YSZ} / \mathrm{CeO}_{2} / \mathrm{Ni}$ tape as determined by electron back-scattered patterns (EBSP) is shown in Fig. 2. A YSZ layer has proven useful in alleviating cracking of the oxide layers that often occurs due to the thermal expansion mismatch between the Ni substrate and the oxides. Figure 2(a) shows an image of a typical film grown by PLD. The location of the grain boundaries from the $\mathrm{Ni}$ grains are clearly evident in the 
morphology due to thermal grooving of the $\mathrm{Ni}$ during annealing. The horizontal and vertical lines are residual cracks that appear along the (110) planes in the $\mathrm{CeO}_{2}$ layer. Figure 2(b) is a shaded map of the local texture determined with EBSP in which a change in crystal orientation greater that $1^{\circ}$ is indicated by a change in shading. Within each $\mathrm{Ni}$ grain, the orientation of the $\mathrm{CeO}_{2}$ and $\mathrm{YSZ}$ grains is uniform to within $1^{\circ}$, which is consistent with epitaxy of the oxides on the (001) Ni surface. Figure 2(c) is a similar plot in which the point-to-point orientation criterion for shade change is $5^{\circ}$. For the region shown, the $\mathrm{Ni}$ grains are textured to within $5^{\circ}$, with this texture reproduced in the oxide buffer layers. This figure clearly illustrates that the orientation in the Ni substrate can be reproduced in the subsequent buffer layers.

The $\theta-2 \theta$ x-ray diffraction (XRD) scan of a $\mathrm{YBCO} / \mathrm{YSZ} / \mathrm{CeO}_{2} / \mathrm{Ni}$ composite structure in Fig. 3 shows that the $\mathrm{CeO}_{2}$ and YSZ buffer layers, as well as the YBCO layer, are (001)-oriented relative to the surface normal. The XRD rocking curve through the $\mathrm{Ni}(002)$ peak shown in Fig. 4 reveals a significant amount of structure, due to the coarse-grained nature of the rolled-textured substrate, yielding an out-of-plane FWHM of $\sim 6^{\circ}$ for the sample considered. Subsequent rocking curves through the (002) peaks for the $\mathrm{CeO}_{2}$ and $\mathrm{YSZ}$ layers indicate an out-of-plane FWHM of $5.5^{\circ}$ and $5^{\circ}$, respectively. Some structure is also observed in these rocking curves, reflecting the good epitaxial relationship between the oxides and the underlying Ni grains. A significant narrowing in the out-of-plane texture is observed for the YBCO when 
deposited directly on the YSZ layer, with the rocking curve through the (005) YBCO peak yielding an out-of-plane FWHM of only $1^{\circ}$. This improvement in the out-of-plane alignment relative to the underlying $\mathrm{Ni}$ results from the low (001) surface energy and the anisotropic film growth nature commonly observed for YBCO. Also shown is the in-plane crystallographic alignment of the epitaxial $\mathrm{YBCO} / \mathrm{YSZ} / \mathrm{CeO}_{2} / \mathrm{Ni}$ structure as determined by XRD $\phi$-scans through the YBCO (226), YSZ (202), $\mathrm{CeO}_{2}$ (202), and $\mathrm{Ni}(222)$ peaks. The inplane FWHM for all of the layers is $\sim 6.8^{\circ}$, indicating excellent epitaxy of the oxide layers with the biaxially textured metal. If the grain-to-grain misorientation angles are uncorrelated with a normal distribution, $\sim 90 \%$ of the $\mathrm{Ni}$ grains have in-plane misorientation angles of $7^{\circ}$ or less for this sample [17]. Note that the in-plane (100) $\mathrm{CeO}_{2}$ and $\mathrm{YSZ}$ principal crystallographic axes are rotated $45^{\circ}$ relative to the in-plane $(100) \mathrm{Ni}$ axis, whereas the a- and b- axes of the YBCO are rotated $45^{\circ}$ with respect to the $\mathrm{YSZ}$ axes, all of which are in agreement with near-coincidence site lattice models [18].

One important factor to consider for the $\mathrm{YBCO} / \mathrm{YSZ} / \mathrm{CeO}_{2} / \mathrm{Ni}$ architecture involves the in-plane orientation of the YBCO films on the YSZ layers. Due to a large lattice mismatch, two distinct in-plane orientations of $\mathrm{YBCO}$ on (100) YSZ are often observed. The presence of two in-plane orientations leads to $45^{\circ}$ grain boundaries and a subsequent reduction in $J_{c}$ : Previous work has shown that various oxide buffer layers, including $\mathrm{CeO}_{2}$, can significantly suppress one of these orientations [19]. By growing $\mathrm{YBCO} / \mathrm{CeO}_{2} / \mathrm{YSZ} / \mathrm{CeO}_{2} / \mathrm{Ni}$ structures with a thin $(\sim 200 \AA) \mathrm{CeO}_{2}$ cap layer on 
the YSZ, improvement in the reproducibility of $J_{c}$ has been observed, with YBCO films routinely obtained on $\mathrm{RABiTS}^{\mathrm{TM}}$ substrates with only one inplane orientation.

Superconducting Transport Properties

Using pulsed-laser deposition, c-axis oriented, in-plane aligned YBCO has been obtained on RABiTS ${ }^{\mathrm{TM}}$ substrates with $T_{c} \sim 87-90 \mathrm{~K}$ and $J_{c}(77 \mathrm{~K})$ as high as $1.4 \mathrm{MA} / \mathrm{cm}^{2}$ for $0.5 \mu \mathrm{m}$ thick films. Figure 5 shows $J_{c}(77 \mathrm{~K})$ as a function of magnetic field applied parallel to the $\mathrm{YBCO} \mathrm{c}$-axis for a film deposited on a RABiTS $^{\mathrm{TM}}$ substrate. For this film, $J_{c}(77 \mathrm{~K}, \mathrm{H}=0) \sim 1.2 \mathrm{MA} / \mathrm{cm}^{2}$. The $J_{\mathcal{c}}$ behavior for the YBCO films on the rolled-textured $\mathrm{Ni}$ tapes is comparable with that observed for epitaxial films on oxide single crystals, which is consistent with the absence of high angle grain boundaries in the YBCO film. In addition, $\mathrm{YBCO}$ films on $\mathrm{RABiTS^{ \textrm {TM } }}$ substrates typically exhibit very strong flux pinning as indicated by the field dependence of $J_{c}$. At high fields, these films can be superior to epitaxial films on single crystal (001) $\mathrm{SrTiO}_{3}$ as observed in Fig. 5 for $\mathrm{H}>3$ Tesla. For comparison, we also indicate the $J_{c}(77$ $\mathrm{K}, \mathrm{H}$ ) behavior for epitaxial Bi-2212 and TlBi-1223 [20] films on single crystal substrates to illustrate the performance advantage of $\mathrm{YBCO}$ on RABiTS ${ }^{\mathrm{TM}}$ relative to other HTS systems.

\section{Engineering Issues for RABiTS-Based HTS Conductors}

The use of thinner (001) Ni tapes holds the promise of achieving high engineering critical current densities $\left(J_{e}\right)$ with 1-2 $\mu \mathrm{m}$ thick YBCO films. 
Previous results have shown that $\mathrm{Ni}$ could be rolled-textured to a thickness of $\sim 25 \mu \mathrm{m}$ with in-plane and out-of-plane texture similar to that obtained for 125 $\mu \mathrm{m}$ thick $\mathrm{Ni}$ tapes. Initial efforts have focused on the epitaxial growth of the $\mathrm{YBCO} / \mathrm{YSZ} / \mathrm{CeO}_{2}$ oxide architecture on $25 \mu \mathrm{m}$-thick $\mathrm{Ni}$ tapes using pulsedlaser deposition. Four-circle x-ray diffraction for the $\mathrm{YBCO} / \mathrm{YSZ} / \mathrm{CeO}_{2}$ oxide architecture on $25 \mu \mathrm{m} \mathrm{Ni}$ shows that the oxide layers are epitaxial and inplane aligned with respect to the Ni substrate. The best result achieved to date with $25 \mu \mathrm{m}$ thick Ni substrates is $J_{c}(77 \mathrm{~K}) \sim 200 \mathrm{kA} / \mathrm{cm}^{2}$, yielding an engineering $J_{c}$ of $11.5 \mathrm{KA} / \mathrm{cm}^{2}$. Significantly higher values for $J_{c}$ are anticipated as superior procedures for handling these relatively thin metal tapes are developed. Future efforts will also focus on understanding the effect of substrate thickness on the conductor properties, particularly when considering the difference in thermal expansion for the oxides layers and the thin Ni tape.

Another important aspect for any HTS wire technology involves the degree to which the conductor can be bent without damaging the conductor. To address this issue, bend strain tolerance measurements of $J_{c}$ have been performed on RABiTS ${ }^{\mathrm{TM}}$ structures. The bend strain tolerance for two YBCO/buffer layers/Ni tapes measuring tensile and compressive strain tolerance is shown in Fig. 6. For both of these samples, the buffer layers and YBCO were deposited using PLD. The samples were placed in strain by deforming the tapes over a selected radius of curvature at room temperature. The strain is then removed (sample curvature is removed) for $J_{c}$ 
measurement in liquid nitrogen. Each data point in the plot represents bending, straightening and thermal cycling. The initial $J_{c}(77 \mathrm{~K})$ for each sample was greater than $0.5 \mathrm{MA} / \mathrm{cm}^{2}$. For compressive strain, significant degradation of $J_{c}$ was not observed until the bend strain exceeded $0.7 \%$, at which point failure was catastrophic. Scanning electron microscopy images of this sample after strain tolerance measurements indicate the presence of long cracks that extend a significant fraction of the sample width. The observation of long cracks in the YBCO film is consistent with the catastrophic failure that was observed in the behavior of $J_{c}$. For the case of tensile strain, a significant decrease in $J_{c}$ with applied strain began at $\sim 0.3 \%$. In contrast to the behavior observed for compressive strain, failure of the sample with tensile strain was more gradual, with complete failure $J_{c}=0$ ) observed for a strain of $\sim 0.5 \%$. The SEM micrograph for this sample shows an array of short fracture lines with the density of cracks higher for the tensile strain than for the sample placed in compression. The appearance of short cracks is consistent with a gradual failure of the film for tensile strain. Future efforts will continue to focus on the effects of specific multilayer oxide architectures on the strain tolerance.

\section{Microstructure of Epitaxial YBCO / Buffer Layers / Ni}

A fundamental understanding of the epitaxial oxide / metal microstructure is crucial in efforts to effectively characterize and further improve the performance of RABiTS ${ }^{\text {TM }}$-based HTS conductors. Microstructural issues that need to be addressed include microcracking, secondary phase nucleation, 
film growth over Ni grain boundary grooves, and substrate oxidation. The scanning electron microscopy image of a $\mathrm{YSZ} / \mathrm{CeO}_{2} / \mathrm{Ni}$ sample shown in Fig. 7 illustrates some of these features. The grooves at the $\mathrm{Ni}$ grain boundaries may impact the nucleation of the $\mathrm{CeO}_{2}$ on the $\mathrm{Ni}$ surface. One must also consider the $\mathrm{YBCO}$ growth across this macroscopic surface feature. In addition, the microcracks that often appear in the $\mathrm{CeO}_{2}$ layer must be reduced or eliminated in order to maximize the ability of the HTS film to carry currents. Note also the appearance of $\mathrm{NiO}$ rods that grow through pinholes in the buffer layers. The presence of these outgrowths provide a source for local nickel contamination of the YBCO film as well as strain in the buffer layer that could lead to additional cracking and perhaps delamination.

Microstructural characterization using cross-section transmission electron microscopy (TEM) has been performed on several YBCO/buffer layer/Ni structures. Figure 8 shows results from a cross-section TEM image of a $\mathrm{YBCO} / \mathrm{YSZ} / \mathrm{CeO}_{2} / \mathrm{Ni}$ sample. This cross-section reveals each of the oxide layers and includes a grain boundary that originates from the Ni substrate. There are several features of interest in this micrograph. First, significant oxidation of the nickel substrate is evident at the $\mathrm{Ni} / \mathrm{CeO}_{2}$ interface with a $\mathrm{NiO}$ layer thickness of $100-400 \AA$. It is assumed that this $\mathrm{NiO}$ layer forms after the nucleation of (100) $\mathrm{CeO}_{2}$ on the (100) Ni surface. The formation of a $\mathrm{NiO}$ layer at the metal/oxide layer interface should have significant influence on the mechanical and adhesive properties of the film due to stresses related to the volume change with the oxidation of $\mathrm{Ni}$ at the buried interface. High 
resolution TEM of the $\mathrm{NiO} / \mathrm{Ni}$ and $\mathrm{NiO} / \mathrm{CeO}_{2}$ interfaces shown in Fig. 9 shows that the $\mathrm{NiO}$ is epitaxial with a $45^{\circ}$ rotation of the $\mathrm{CeO}_{2}$ principle axes with respect to the $\mathrm{NiO}$.

The cross-section of the $\mathrm{CeO}_{2}$ layer shown in Fig. 10 reveals three distinct microstructures that correspond to changes in the film growth conditions during the initial nucleation and subsequent growth of the $\mathrm{CeO}_{2}$ layer. The initial $\mathrm{CeO}_{2}$ is deposited in the presence of $\mathrm{Ar} / \mathrm{H}_{2}$ as pointed out earlier. This results in a somewhat uniaxial microstructure. After the nucleation of $\sim 300$ $\AA$, the $\mathrm{Ar} / \mathrm{H}_{2}$ background gas is evacuated while continuing to deposit an additional $500 \AA$ of $\mathrm{CeO}_{2}$. This growth in a background pressure of $\sim 10^{-5}$ Torr results in a more columnar microstructure. This is then followed by growth in an oxygen pressure of $4 \times 10^{-4}$ Torr which again results in a more uniaxial microstructure.

The microstructure of the YSZ layer is distinctly columnar as indicated in Fig. 11. Some evidence for a $\mathrm{BaZrO}_{3}$ layer at the $\mathrm{YBCO} / \mathrm{YSZ}$ interface is observed, although the thickness of this interaction layer is minimal. One interesting issue regards how the groove at the $\mathrm{Ni}$ grain boundary is accommodated in the various layers. From Fig. 8, we observed that the morphology of the groove is reproduced in each of the oxide layers. Figure 11 shows that the orientation of the columnar microstructure within the YSZ layer follows the morphology of the groove. With this macroscopic change in local microstructure, one might anticipate significant disruption of the YBCO epitaxy across this feature based on the tendency of YBCO to align the c-axis 
perpendicular to the growing surface, as well as the behavior of YBCO epitaxy across etched steps on single crystal surfaces. This could prove detrimental to transport with the local formation of large angle grain boundaries across the groove. Fortunately, this does not appear to be the case. Deep within the groove, the epitaxy of YBCO appears to be disrupted. However, the YBCO grains adjacent to the groove apparently grow over the grooved region. Note that the grain boundary location, while effectively translated vertically within the buffer layer architecture, has shifted laterally to the edge of the groove. An explanation for this observation is that the $\mathrm{YBCO}$ grains that nucleate on flat regions of the substrate dominate film growth leading to overgrowth over the grooved region. This is consistent with previous bicrystal experiments in which significant meandering of the YBCO grain boundary is observed with respect to the location of the grain boundary in the underlying bicrystal substrate.

Plan-view Z-contrast scanning transmission electron microscopy (Z-STEM) provides additional insight regarding the atomic structure of the YBCO grain boundaries for films deposited on these substrates. Figure 12 shows an atomic resolution Z-STEM image of a typical YBCO low-angle grain boundary that is commonly observed in c-axis oriented YBCO films deposited on rolled-textured (001) Ni tape. These low-angle grain boundaries in epitaxial YBCO film on RABiTS ${ }^{\mathrm{TM}}$ originate from the grain structure in the biaxially-textured (001) $\mathrm{Ni}$ tape. As seen in the figure, the atomic structure of the grain boundary closely resembles that observed for YBCO films on $\mathrm{SrTiO}_{3}$ 
bicrystals, consisting of a "clean" boundary with a regular array of dislocation cores. From the micrograph, there is no evidence for impurity phases, $\mathrm{NiO}$, or misoriented $\mathrm{YBCO}$ grains at the boundary. This is consistent with the relatively high critical currents that are observed for $\mathrm{YBCO} /$ buffer layers/(001) biaxially textured Ni structures.

\section{Conclusion}

The use of biaxially-textured substrates for HTS film growth opens up an array of large-area HTS film applications, including the synthesis of superconducting YBCO tapes for high-field applications at $77 \mathrm{~K}$. Epitaxial YBCO films with $J_{c}>1 \mathrm{MA} / \mathrm{cm}^{2}$ at $77 \mathrm{~K}$ have been realized on rolled-textured $\mathrm{Ni}$ tapes. A better understanding of metal oxide/metal epitaxial systems is needed in order to fully characterize and further improve the performance of this coated-conductor architecture in progressing from laboratory-scale samples to useful conductor lengths.

\section{ACKNOWLEDGEMENTS}

The authors would like to thank P. H Fleming for assistance in substrate preparation and film patterning. This research was sponsored by the Oak Ridge National Laboratory, managed by Lockheed Martin Energy Research Corp., for the U.S. Department of Energy, under contract DE-AC05-96OR22464. 


\section{REFERENCES}

1. J. W. Ekin, K. Salama, V. Selvamanickam, Nature 350, 26 (1991).

2. D. T. Shaw, MRS Bull. 17, 39 (1992); E. E. Hellstrom, ibid. 17, 45 (1992).

3. J. M. Phillips, J. Appl. Phys. 79,1829 (1996).

4. D. Dimos et al., Phys. Rev. Lett. 61, 1653 (1988).

5. D. K. Christen et al., in AIP Conf. Proc., Superconductivity and its Applications 273, 24 (1993).

6. M. Rupp, A. Gupta, C. C. Tsuei, Appl. Phys. Lett. 67, 291 (1995).

7. Y. Iijima, N. Tanabe, O. Kohno, and Y. Ikeno, Appl. Phys. Lett. 60, 769 (1992).

8. X. D. Wu et al., Appl. Phys. Lett. 67, 2397 (1995).

9. K. Hasegawa et al., Proc. of the 9th Intl. Symp on Superconductivity (ISS"96), Oct. 1996, Sapporo, Japan.

10. M. Fukutomi et al., Physica C 231, 113 (1994).

11. H. Makita, S. Hanada, O. Izumi, Acta Metall. 36, 403 (1988).

12. D. P. Norton et al., Science 274, 755 (1996)

13. A. Goyal, et al., Appl. Phys. Lett. 69, 1795 (1996).

14. N. N. Khoi, W. W. Smeltzer, J. D. Embury, J. Electrochem. Soc. 122, 1495 (1975).

15. Q. He et al., Physica C 275, 155 (1997).

16. M. Paranthaman et al., Physica C 275, 266 (1997).

17. E. D. Specht et al., Physica C 226, 76 (1994). 
18. J. D. Budai, R. T. Young, B. S. Chao, Appl. Phys. Lett. 62, 1836 (1993).

19. D. K. Fork, S. M. Garrison, M. Hawley, and T. H. Geballe, J. Mater. Res. 7, 1641 (1992).

20. L.P. Guo, Z. F. Ren, J.Y. Lao, J. H. Wang, D. K. Christen, C.E. Klabunde, J.D. Budai, Physica C 277, 13 (1997). 
Figure Captions

Figure 1. Schematic of the IBAD and RABiTS ${ }^{\mathrm{TM}}$ processes for fabricating biaxially-textured coated HTS conductors.

Figure 2. Electron baskscattered images of epitaxial $\mathrm{YSZ} / \mathrm{CeO}_{2}$ epitaxial layers on rolled-textured Ni showing the morphology (a) as well as the crystallographic texture of a typical RABiTS ${ }^{\mathrm{TM}}$ sample. The point-to-point shading criteria for a change in grey-scale shading is (b) $1^{\circ}$ and (c) $5^{\circ}$.

Figure 3. X-ray diffraction $\theta-2 \theta$ scan of a $\mathrm{YBCO} / \mathrm{YSZ} / \mathrm{CeO}_{2} / \mathrm{Ni}$ structure showing the c-axis orientation of the buffer and YBCO layers.

Figure 4. X-ray diffraction scans showing the in-plane and out-of-plane texture for a typical $\mathrm{YBCO} / \mathrm{YSZ} / \mathrm{CeO}_{2} / \mathrm{Ni}$ sample.

Figure 5. The magnetic field dependence of $J_{c}(77 \mathrm{~K})$ for a $0.6 \mu \mathrm{m}$ thick YBCO film on a $\mathrm{YSZ} / \mathrm{CeO}_{2} /$ rolled Ni substrate. The $J_{c}(77 \mathrm{~K}, \mathrm{H})$ behavior for YBCO on (001) $\mathrm{SrTiO}_{3}$ as well as for epitaxial Bi-2212 and TlBi-1223 films is also shown for comparison.

Figure 6. Strain tolerance data for $\mathrm{YBCO}$ on $\mathrm{RABiTS}^{\mathrm{TM}}$ samples measured at $77 \mathrm{~K}$.

Figure 7. A scanning electron microscopy image of a $\mathrm{YSZ} / \mathrm{CeO}_{2} /$ rolled $\mathrm{Ni}$ sample indicating the location of $\mathrm{Ni}$ grain boundaries, micorcracks due to thermal expansion mismatch, and $\mathrm{NiO}$ protrusions. 
Figure 8. A cross-section transmission electron microscopy image of a $\mathrm{YBCO} / \mathrm{YSZ} / \mathrm{CeO}_{2} /$ rolled $\mathrm{Ni}$ sample. The total $\mathrm{CeO}_{2}$ layer thickness is approximately $900 \mathrm{~nm}$.

Figure 9. A high-resolution transmission electron microscopy image of the $\mathrm{Ni} / \mathrm{NiO}$ and $\mathrm{NiO} / \mathrm{CeO}_{2}$ epitaxial interfaces.

Figure 10. A cross section transmission electron microscopy image of the $\mathrm{CeO}_{2}$ layer, with the varying microstructure corrsponding to background ambients of (a) $200 \mathrm{mTorr} \mathrm{Ar} / \mathrm{H}_{2}$ (b) vacuum, or (c) $4 \times 10^{-4}$ Torr $\mathrm{O}_{2}$. Figure 11. Cross-section transmission electron microscopy image of the YSZ layer showing a columnar microstructure and location of the grain boundary.

Figure 12. A plan-view Z-contrast scanning transmission electron microscopy image of a YBCO film on a RABiTS ${ }^{\mathrm{TM}}$ substrate showing the grain boundary. 

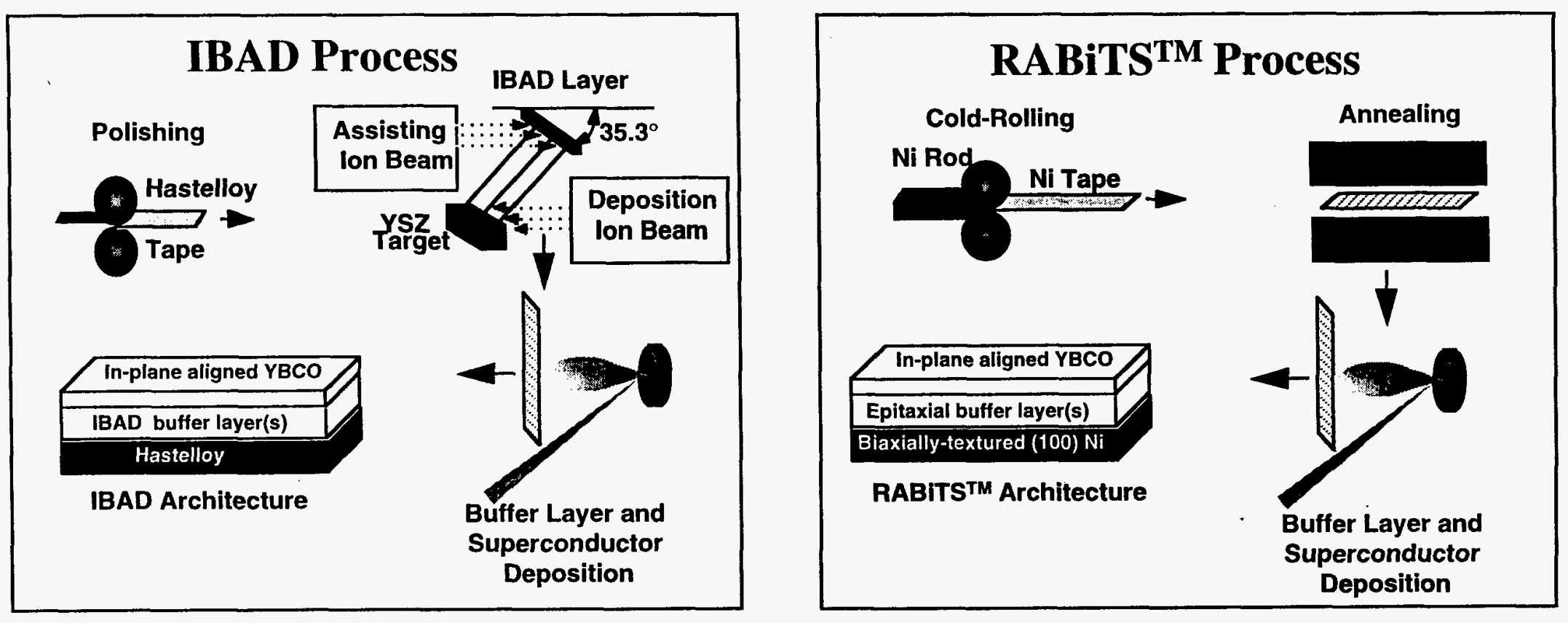

Figure 1 
乙

\section{m 81}
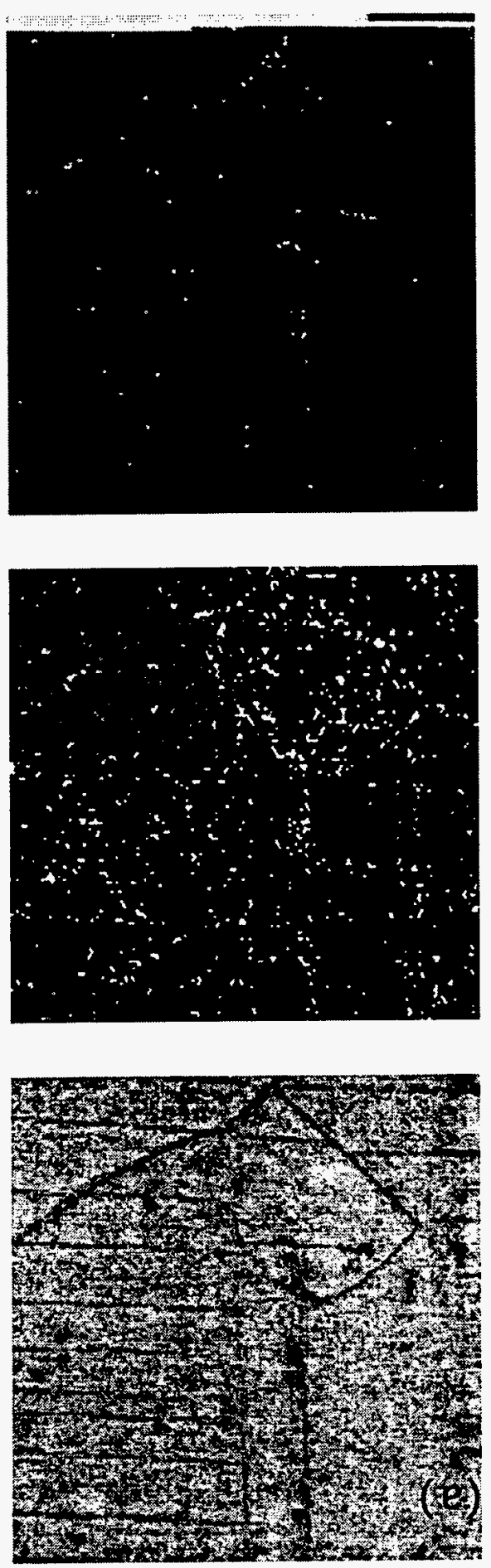


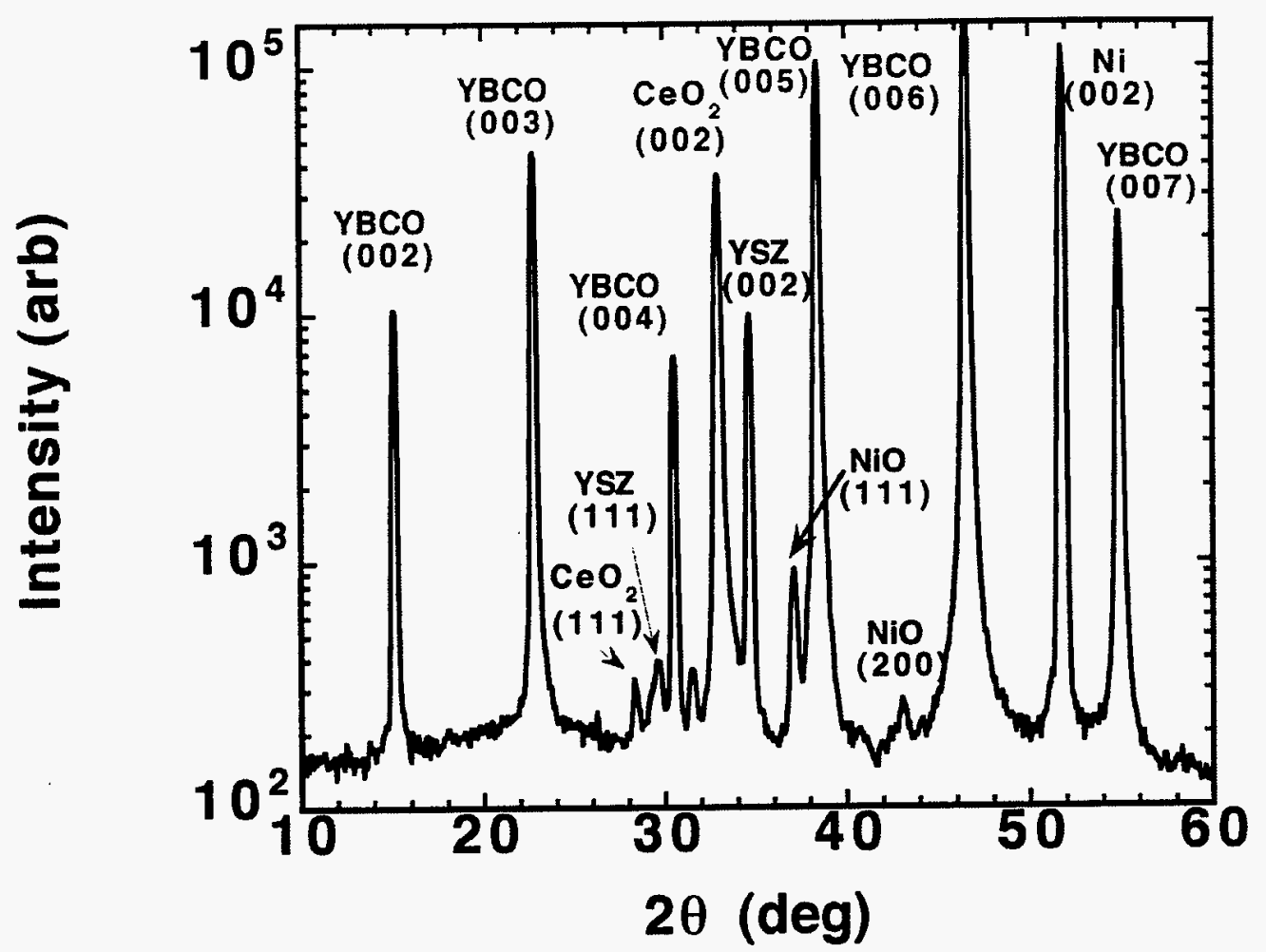

Figure 3 


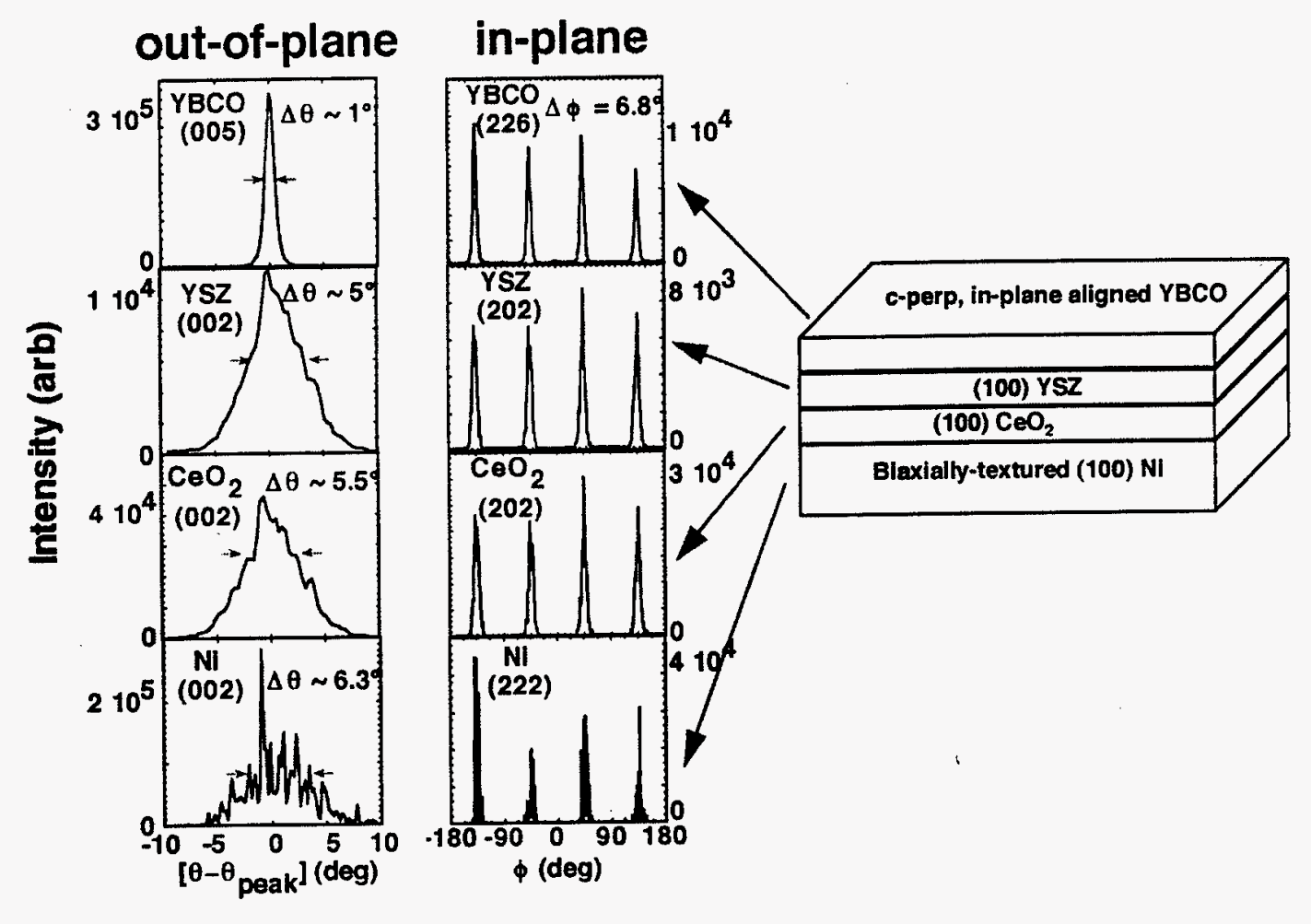

Figure 4 


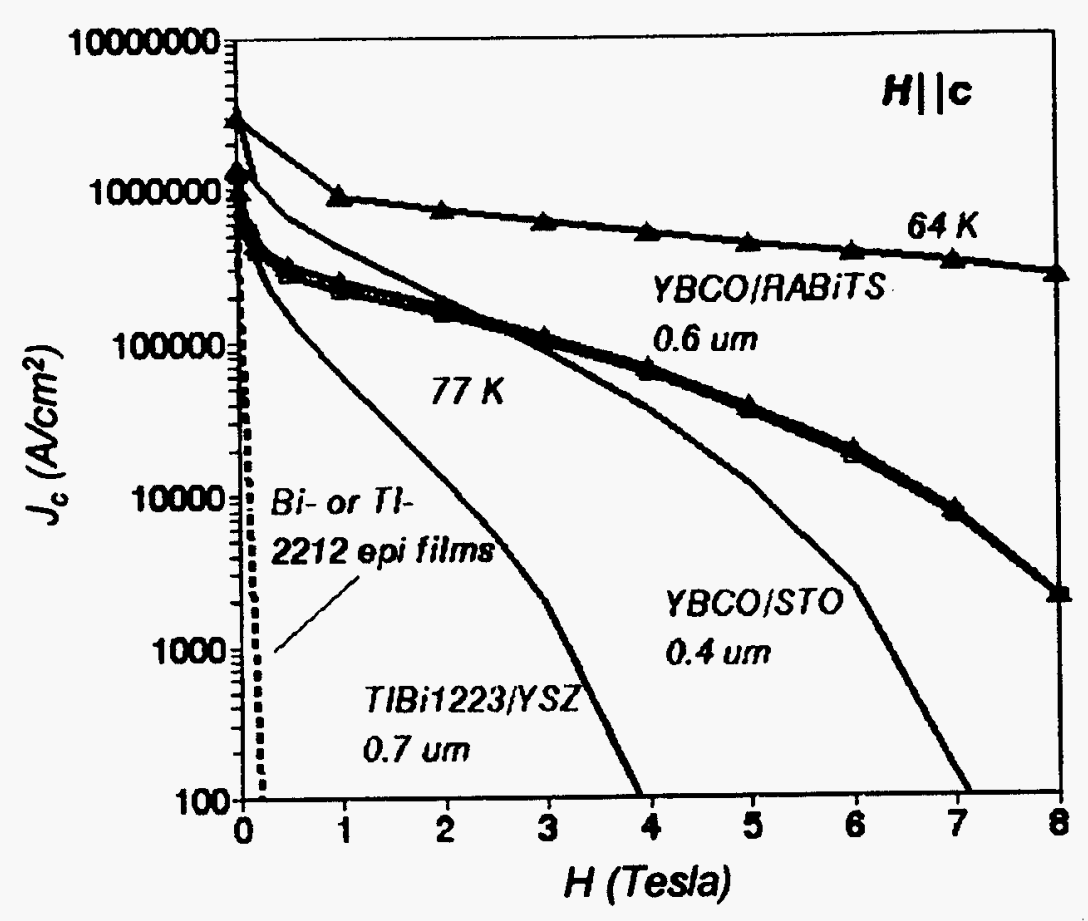

Figure 5 

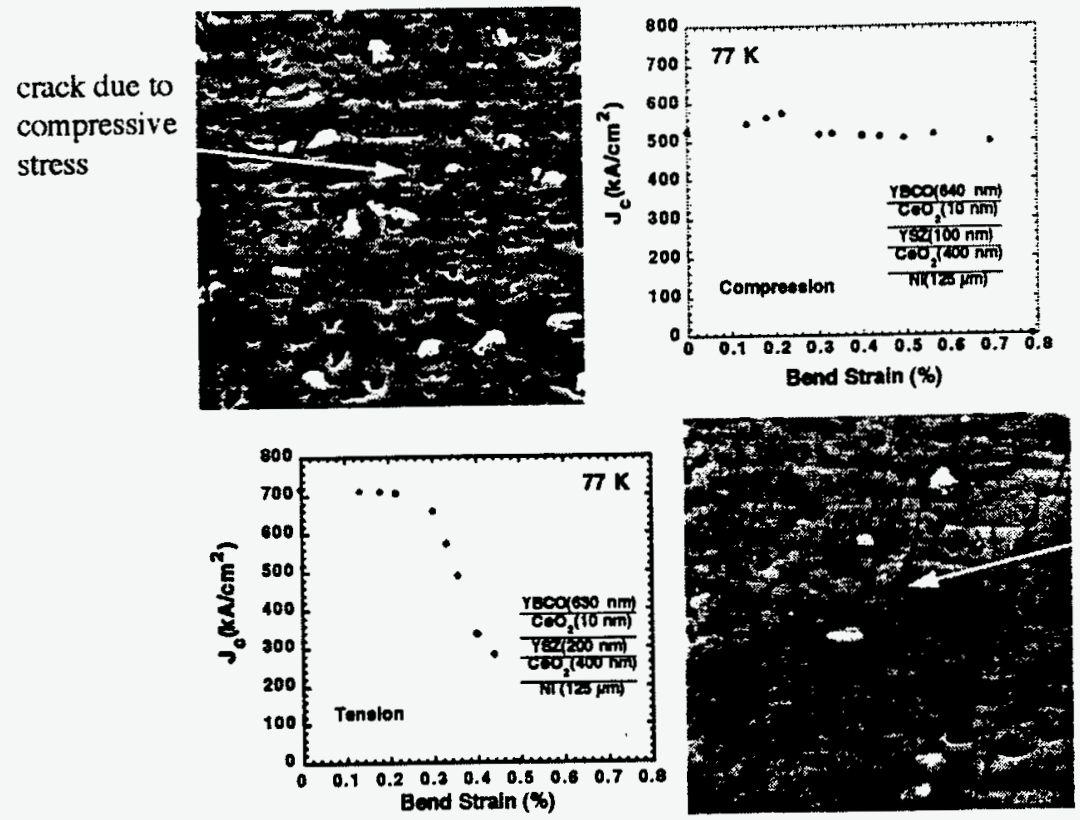

crack due to tensile stress 


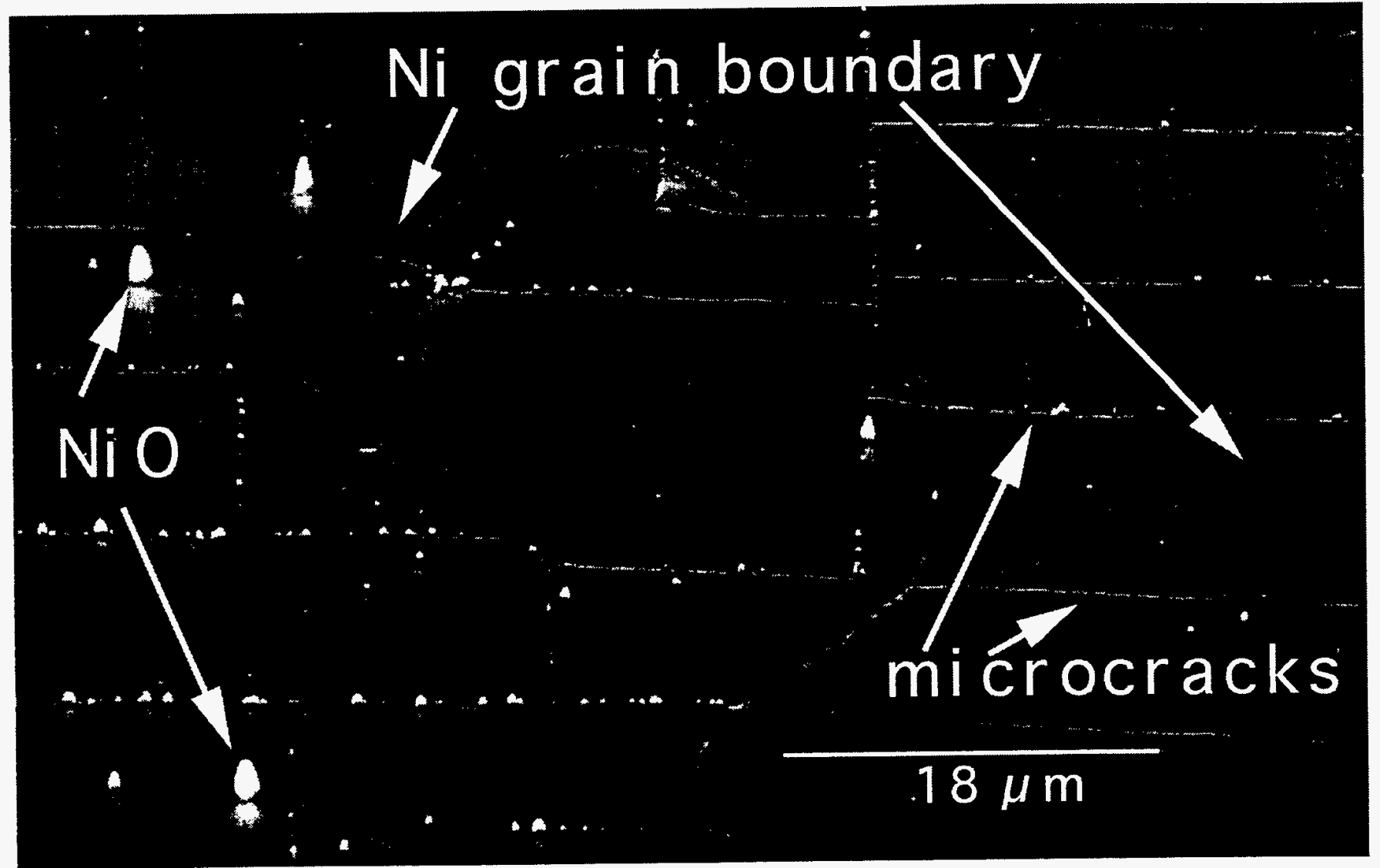

Figure 7 
YBCO

YSZ

$\mathrm{CeO}_{2}$

$\mathrm{Ni}$
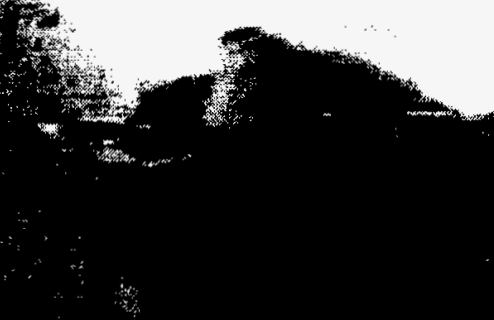

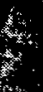

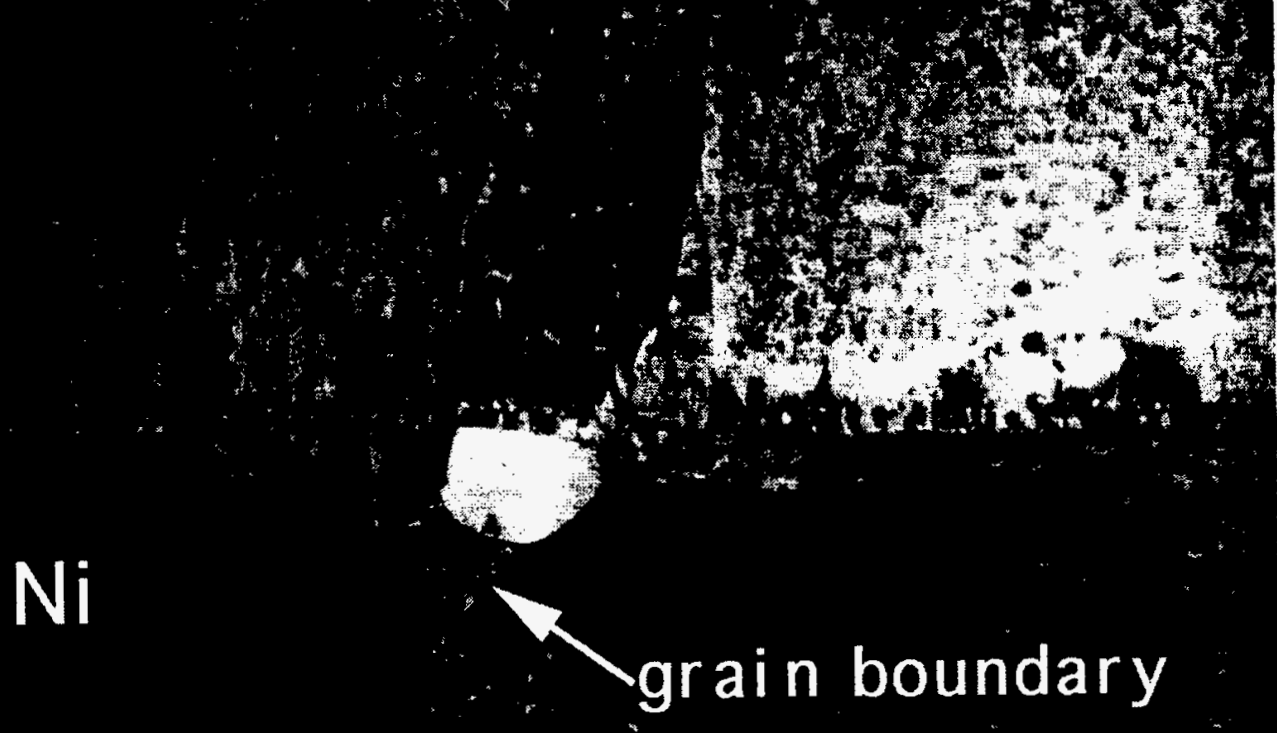

Figure 8 

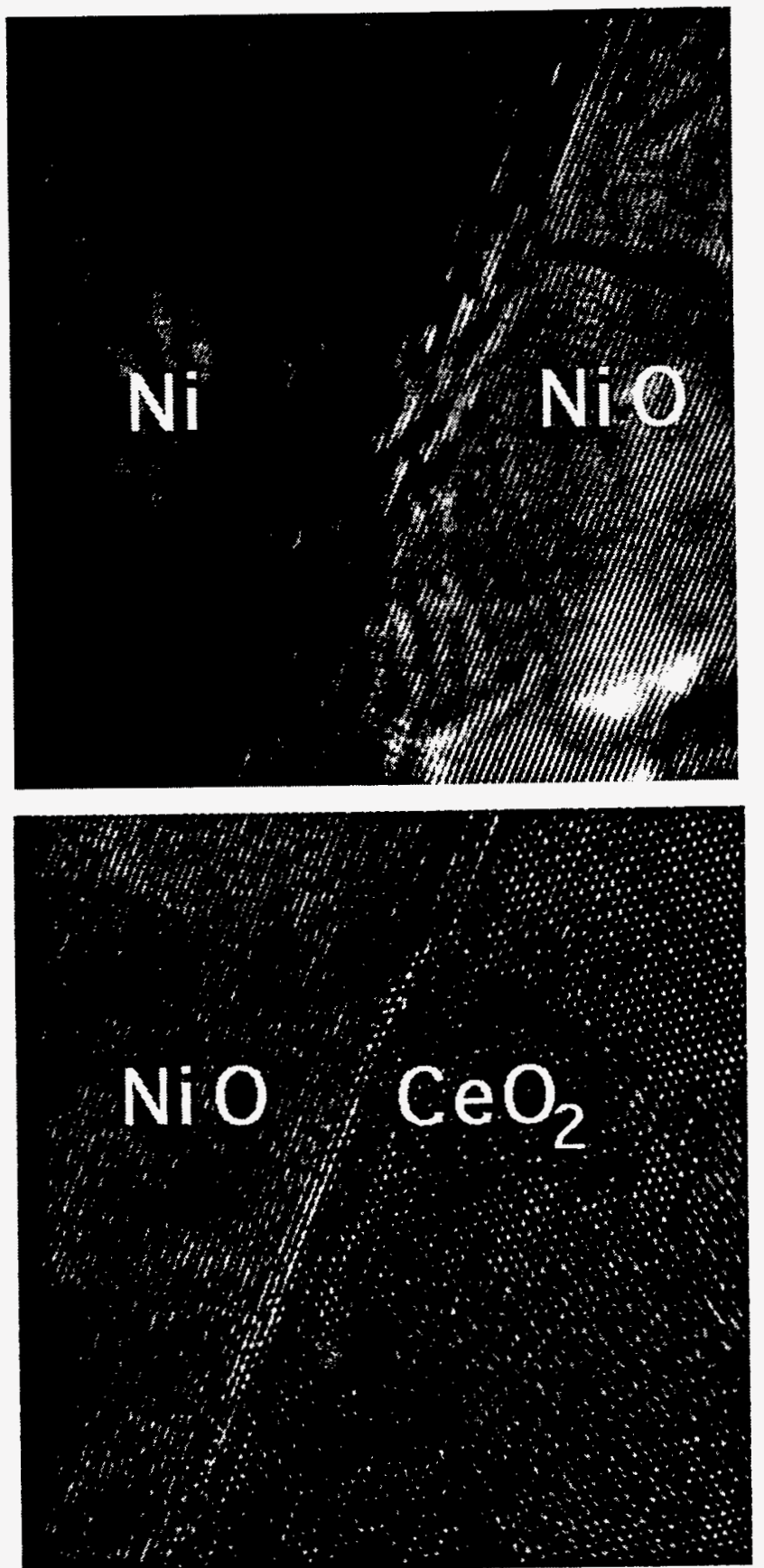

Figure 9 


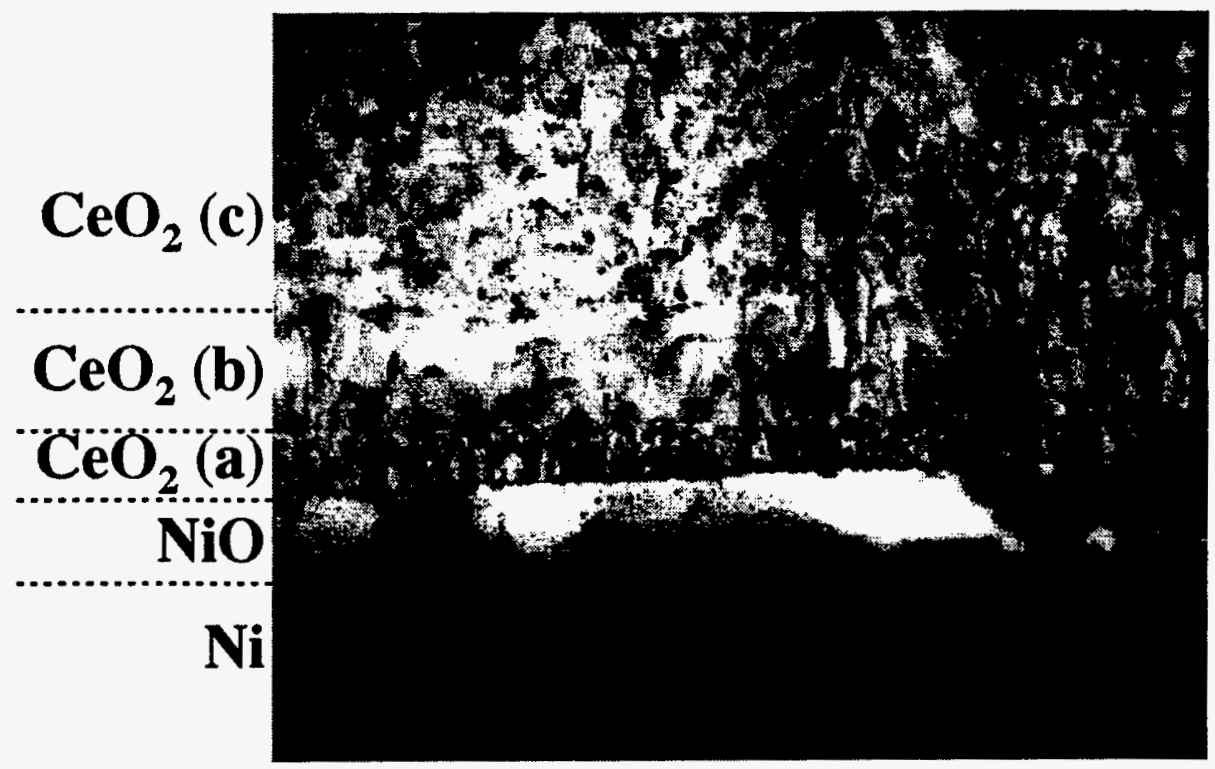

Figure 10 


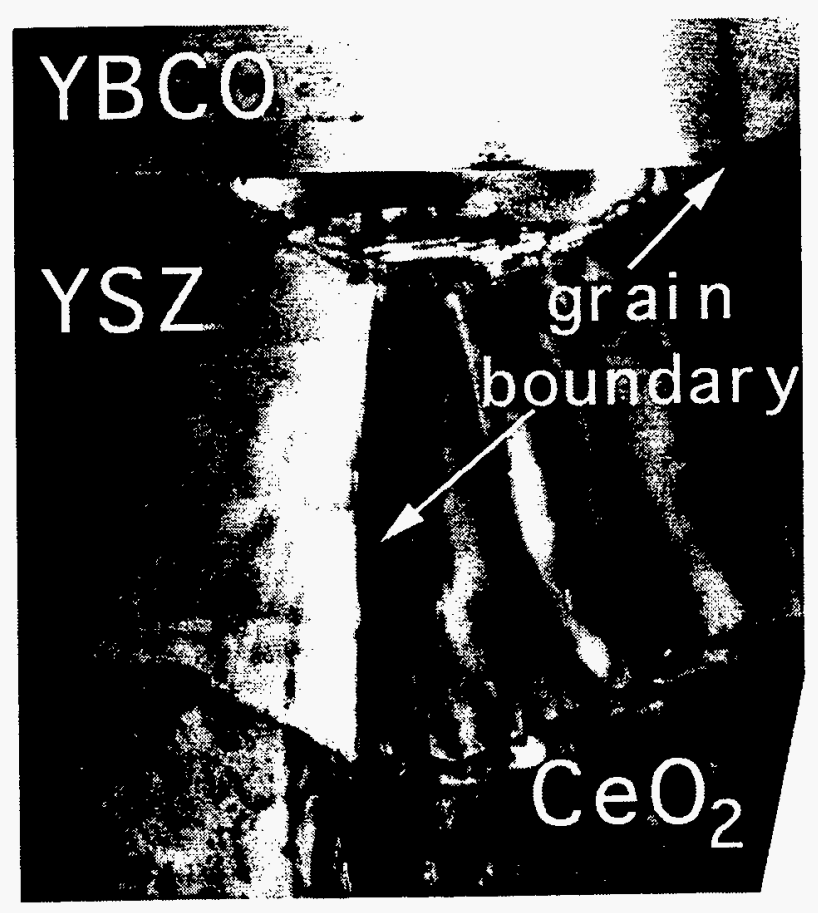

Figure 11 


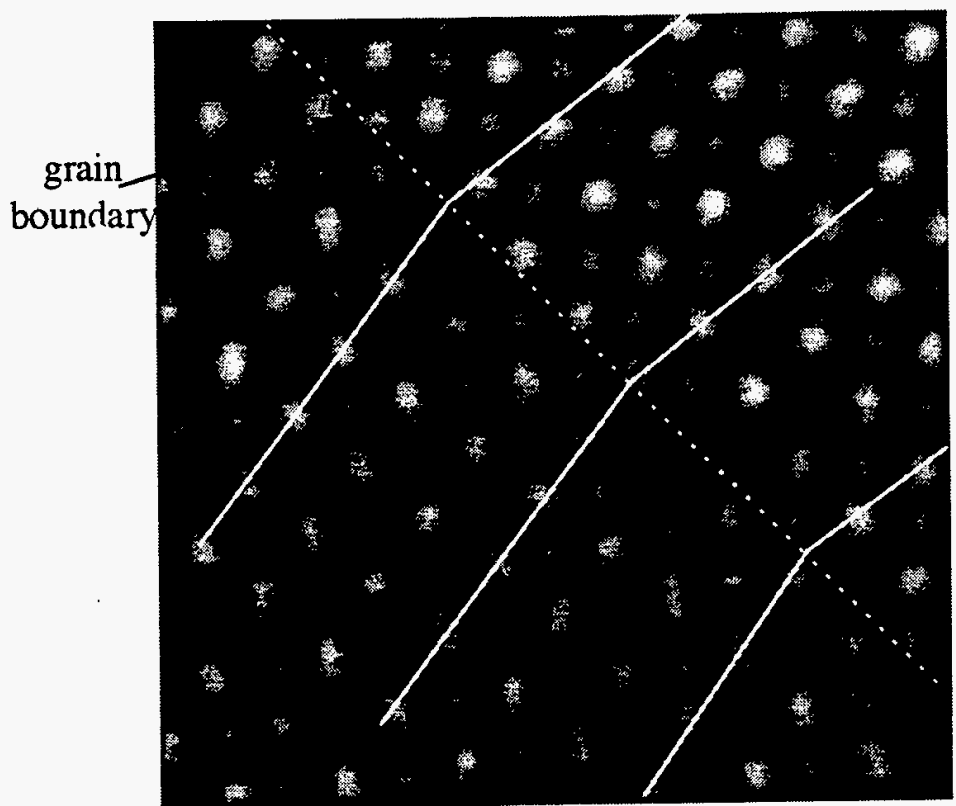

Figure 12 


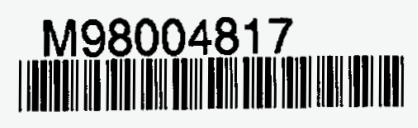

Report Number (14) ORNL/CP-.9752C

Publ. Date (11) 199804
Sponsor Code (18)
UC Category (19) UC-4.04, XOE/ER 